\title{
Impacto ambiental de los residuos sólidos en el distrito de Santa María - Huaura año 2018
}

\section{Environmental impact of solid waste in the Santa Maria District - Huaura Year 2018}

Aparicio Fortunato León Ostos ${ }^{\text {; }}$ Dante Daniel Cruz Nieto1, Yulisa Elizabeth León Salvador Félix Gil Caro Soto, Yasmin Jesús Vélez Chang ${ }^{1}$, José Antonio Legua Cárdenas

\section{RESUMEN}

Objetivo: Caracterizar y determinar la producción percápita de residuos sólidos y su impacto ambiental en el distrito de Santa María, 2018. Material y Métodos: Se analizaron 49 muestras y se determinó que la cantidad total de residuos que recolectaron fueron de 15,906 kg/día; con una producción per cápita de 0,515 kg/habitante por día, con una densidad promedio de 110,48 kg/m³ , con humedad promedio de $38,66 \%$ y que el mayor componente lo constituye la materia orgánica con el 42,77 \%. Resultados: La población evaluada mostraron problemas en cuanto a su actitud para la recolección de los residuos domésticos. El 47,8\% de la población manifestaron que la municipalidad debería de fomentar más programas ambientales. Conclusiones: Las diversas zonas evaluadas del distrito de Santa María presentan problemas en relación a implementación de programas de concientización ambiental para una correcta disposición final de residuos sólidos.

Palabras clave: Residuos sólidos domiciliarios; caracterización; impacto ambiental y concientización

\begin{abstract}
Objective: To characterize and determine the per capita production of solid waste and its environmental impact in the district of Santa María, 2018. Material and Methods: 49 samples were analyzed and it was determined that the total amount of waste collected was $15,906 \mathrm{~kg} / \mathrm{day}$; with a per capita production of $0.515 \mathrm{~kg} /$ inhabitant per day, with an average density of $110.48 \mathrm{~kg} / \mathrm{m} 3$, with an average humidity of $38.66 \%$ and that the largest component is organic matter with $42.77 \%$. Results: The population evaluated showed problems regarding their attitude to the collection of household waste. $47.8 \%$ of the population said that the municipality should encourage more environmental programs. Conclusions: The various areas evaluated in the Santa María district present problems in relation to the implementation of environmental awareness programs for a correct final disposal of solid waste.
\end{abstract}

Keywords: Solid household waste; characterization; environmental impact and awareness

\section{INTRODUCCIÓN}

En el Perú no se utilizan sistemas de tratamiento eficientes de los residuos sólidos, contándose en la mayoría de las ciudades sólo con botaderos a cielo abierto, o realizándose la incineración no controlada de los residuos municipales. Sin embargo, Fuentes, Carpio, Prado y Sánchez (2008), mencionan que se toma como modelos de experiencia de aprendizaje nacional de los casos de la Municipalidad Provincial de Carhuaz, Municipalidad Distrital de Independencia, Ancash y Municipalidad Distrital de Santiago de Surco, Lima.

Dentro de este contexto, la presente investigación permite:

* Obtener una gama de indicadores tanto de los residuos sólidos urbanos del distrito de Santa María, así como de la contaminación ambiental relacionada.

* Contar con alternativas para realizar y ejecutar acciones para preservar el medio ambiente y mejorar la limpieza en beneficio de la población, evitando a la vez la propagación de enfermedades contagiosas.

* Describir metodologías de manejo y tratamiento de residuos sólidos urbanos acondicionadas a los diferentes distritos de la Provincia de Huaura.

* La oportunidad para minimizar costos sobre el mantenimiento del servicio de recolección y disposición final de los residuos sólidos urbanos en el Distrito de Santa María.

Hasta hace pocos años solía decirse que la preocupación ambiental y el desarrollo económico de un país eran contrapuestos, y que una nación en vías de crecimiento tenía que superar una etapa de desarrollo económico, social y político para empezar a preocuparse por el tema ambiental, Gómez, (2013) expone tanto el medio natural como el creado por el hombre mismo, son esenciales para su bienestar y el goce de los derechos humanos fundamentales, como el derecho a la vida.

Pues con estas afirmaciones cabe manifestar que los residuos urbanos abarca los materiales sólidos orgánicos e inorgánicos que se producen en los mismos, lo que se precisa que la fracción inorgánica está conformada en su mayoría por vidrio, latas, aluminio, cartón, papel; la fracción correspondiente a los orgánicos está conformada en su mayoría por residuos de carne de pescado, res, pollo, residuos de vegetales y frutas, comida entre otros.

Los residuos orgánicos se puede emplear para le elaboración de compost como abono orgánico para el mejoramiento físico químico de suelo y aportando nutrientes para el desarrollo de las plantas en áreas verdes. Lo cual es favorable para la gestión ambiental en su disposición final de aprovechamiento como abono orgánico para las hortalizas; puesto que es sostenible y sustentable obteniendo un producto agro ecológico (Cruz, 2018)

Con el fin de precisar que residuos sólidos orgánicos; e inorgánicos que emanan el distrito de Santa María y sus comunidades se realizó este trabajo de investigación, para determinar qué cantidades se obtiene porcentualmente por año y cuál es el impacto ambiental y social que nos espera en un proyección futura. También se determinó su caracterización física y química de dichos materiales reciclados y sus tendencias representadas gráficamente en la disposición del residuos doméstico en el distrito de Santa María.

Para este trabajo de investigación se realizó análisis estadísticos en función a operaciones estadísticas básicas para el procesamiento de datos para lo cual se hizo gráficas y cuadros en base a la información tabulada; estos resultados se interpretaron y concluyeron tal como se refiere en el presente proyecto.

\section{MATERIALY MÉTODOS}

\section{Población}

Están constituidos por los residuos sólidos del distrito de Santa María, generados por los 29,686 habitantes censados al 2009 residentes de 7,251 viviendas censados al año 2007 y proyectados en el presente estudio para el año 2011 en 30,893 habitantes y 8,085 viviendas. distribuidos en las siguientes zonas, Pacocha, Fujimori, San Bartolomé, Luriama y Santa 
María (INEI, 2009-2015),

\section{Muestra}

La determinación del tamaño de la muestra de viviendas necesarias para la realización de la caracterización de residuos sólidos se calculó de acuerdo a lo descrito en la metodología propuesta por el Dr. Kunitoshi Sakurai, publicada por el Centro Panamericano de Ingeniería Sanitaria y Ciencias del Ambiente, CEPIS, tal como se muestra en la siguiente expresión (Cantanhede, et. al. 2005).

Fórmula:

$$
n=\frac{\left(Z_{1 \frac{\alpha}{2}}^{2}\right)(N)\left(\sigma^{2}\right)}{(N-1) E^{2}+\left(Z_{1 \frac{\alpha}{2}}^{2}\right)\left(\sigma^{2}\right)}
$$

Dónde:

$\mathrm{n}$ : Es el tamaño de la muestra $=49$ viviendas

Técnicas de recolección de datos

\section{Aplicación de la encuesta}

Con el fin de obtener información relacionada al número de personas que viven en cada vivienda y además saber sus conocimientos, apreciaciones y sugerencias acerca del manejo de los residuos sólidos en el distrito, se aplicó la encuesta a los moradores de las viviendas de la muestra en cada zona señalada.

\section{RESULTADOS}

Tabla 1

Producción per cápita de los Residuos Sólidos Urbanos en las principales zonas.

\begin{tabular}{lc}
\hline $\begin{array}{c}\text { Zonas del Distrito de } \\
\text { Santa María }\end{array}$ & $\begin{array}{c}\text { Producción per cápita de RS } \\
\text { (kg/persona, por día) }\end{array}$ \\
\hline Pacocha & 0,516 \\
Fujimori & 0,415 \\
San Bartolomé & 0,601 \\
Luriama & 0,470 \\
Santa María & 0,581 \\
Media & 0,515 \\
\hline
\end{tabular}

Fuente: El autor (2018)

Interpretación: En la tabla 1, se determina que en la zona de San Bartolomé se obtuvo 0,601 kg./persona por día, siendo la zona con más producción en referencia a las demás zonas, puesto que hay más concentración de personas que habitan dicholugar.
Tabla 2

Caracterización de los residuos del Distrito de Santa María

\begin{tabular}{|c|c|c|c|}
\hline $\mathrm{N}^{\circ}$ & Materiales segregados & $\begin{array}{l}\text { Total } \\
(\mathrm{Kg})\end{array}$ & $\begin{array}{c}\text { Composición } \\
\text { porcentual }\end{array}$ \\
\hline 1 & $\begin{array}{l}\text { Restos de cocina (1) excepto } \\
\text { huesos }\end{array}$ & 51,20 & 24,75 \\
\hline 2 & Huesos & 1,50 & 0,72 \\
\hline 3 & Restos de jardín (2) & 9,70 & 4,69 \\
\hline 4 & $\begin{array}{l}\text { Restos de servicios higiénicos } \\
\text { (3) }\end{array}$ & 8,30 & 4,01 \\
\hline 5 & Papel blanco tipo bond & 6,00 & 2,90 \\
\hline 6 & Papel periódico & 4,50 & 2,17 \\
\hline 7 & Papel de envoltura & 2,80 & 1,35 \\
\hline 8 & Cartón & 10,20 & 4,93 \\
\hline 9 & Botellas plásticas transparentes & 5,10 & 2,46 \\
\hline 10 & Envases plásticos & 7,70 & 3,72 \\
\hline 11 & Bolsas plásticas & 7,60 & 3,67 \\
\hline 12 & Plásticos en general & 5,40 & 2,61 \\
\hline 13 & Tecnopor y similares & 1,20 & 0,58 \\
\hline 14 & Jebes y artículos de jebe & 1,70 & 0,82 \\
\hline 15 & Botellas y envases de vidrio & 6,00 & 2,90 \\
\hline 16 & Vidrio en general & 5,40 & 2,61 \\
\hline 17 & Lata y tapas de lata & 6,50 & 3,14 \\
\hline 18 & Metales & 1,80 & 0,87 \\
\hline 19 & Pilas & 0,40 & 0,19 \\
\hline 20 & Madera y aserrín & 4,70 & 2,27 \\
\hline 21 & Cuero & 4,10 & 1,98 \\
\hline 22 & Telas, textiles & 5,40 & 2,61 \\
\hline 23 & Material inerte & 46,70 & 22,57 \\
\hline \multirow[t]{2}{*}{24} & Otros & 3,00 & 1,45 \\
\hline & Total & 206,90 & 100,0 \\
\hline
\end{tabular}

Fuente : El autor (2018)

Interpretación: En los segregados no están los restos de jeringas, algodones, medicamentos, gasas y agujas hipodérmicas. 


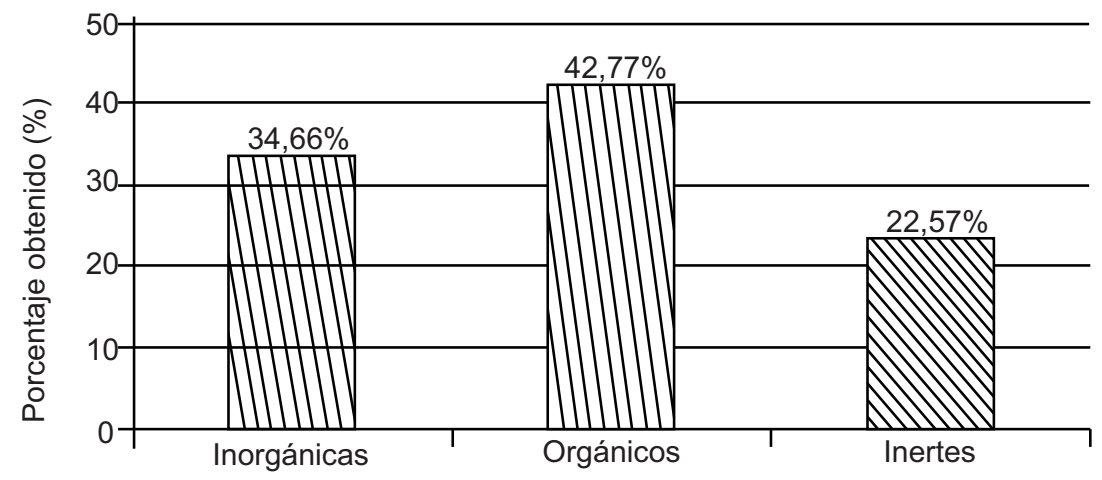

Características de RSU

Figura 1. Determinación de residuos del Distrito de Santa María

En la Figura 1, se aprecia que los residuos orgánicos alcanzan el mayor porcentaje de $42,77 \%$, esto se puede fundamentar con Riofrío, Olivera y Callirgos (1994) expone acerca de que los residuos de alimentos constituyen el componente más grande en las ciudades de los países en vías de desarrollo.
También se basa por Cuello y Tola (1997), manifiestan que en general existe un notable paralelismo entre el grado de desarrollo de una ciudad o sociedad y la composición de los desechos sólidos que generan las ciudades que contienen más pobladores.

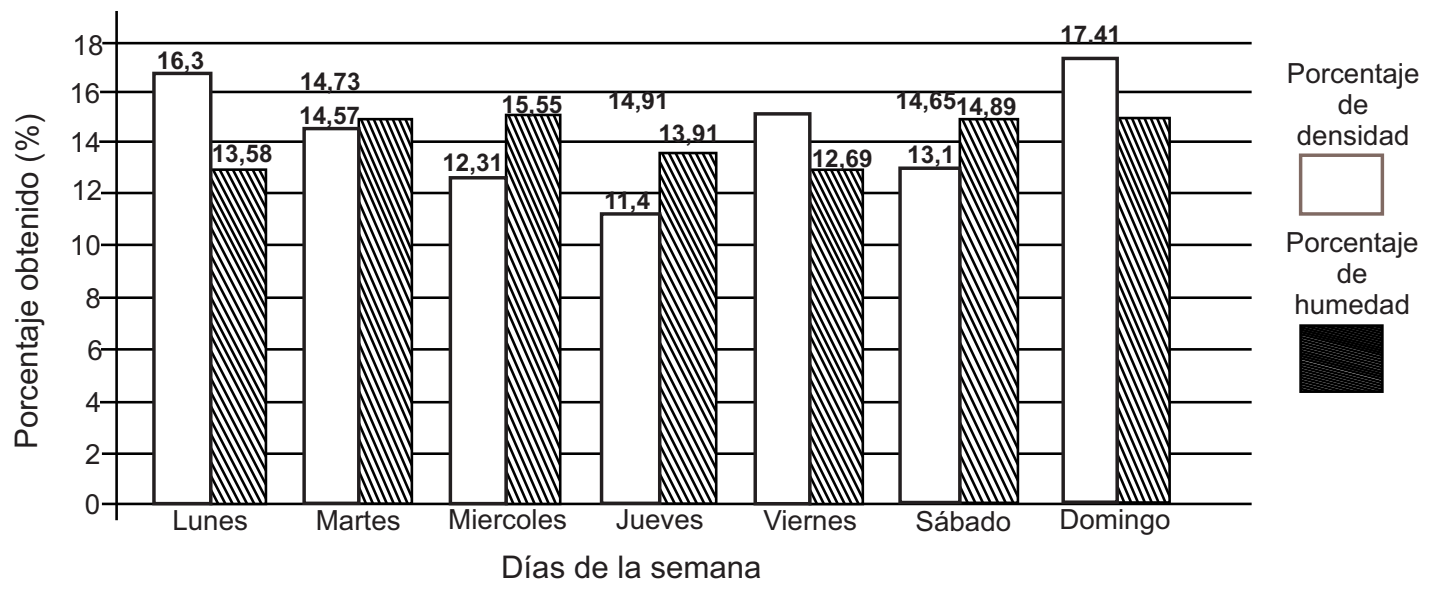

Figura 2. Porcentaje de densidad y porcentaje de residuos en el distrito

En cuanto a la Figura 2 se observa que el domingo alcanza mayor porcentaje de residuos, ya sea por el comercio de fin de semana, esta evaluación se fundamenta con Flores y Villafuerte (2003) quienes señalan que las características físicas de los residuos sólidos varían en función de los hábitos de consumo y del nivel de vida de los generadores. También Rodríguez (1999) explica esta diferencia debido a que la población con mayor posibilidad económica, consume una mayor cantidad de productos envasados que tienen bastante volumen y poco peso.

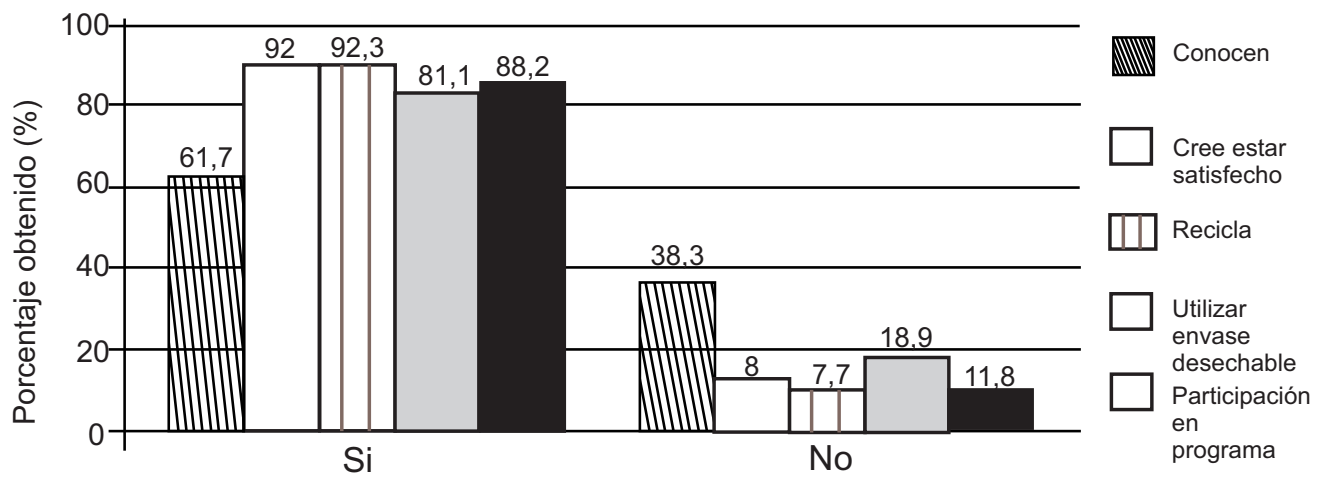

Acciones

Figura 3. Evaluación de opiniones, según sus acciones de dicho distrito

La Figura 3, señala que el mayor porcentaje de la población recicla, su opinión de estar satisfecho, si utiliza envase desechable y si participa en programas de reciclaje, obtuvo el calificativo de "Bueno" el servicio de recolección de residuos prestado por la Municipalidad, este resultado es debido al servicio eficiente por lo que se contrasta con lo indicado por Arroyo, Rivas y Lordinois (1997) afirman que son los sectores de ingresos altos y medios los que se ven favorecidos regularmente con el mejor servicio y en cambio los sectores pobres o marginales sólo cuentan con servicios esporádicos, cuando los tienen. Sin embargo, la población está de acuerdo en que se deberían mejorar ciertas dificultades. 


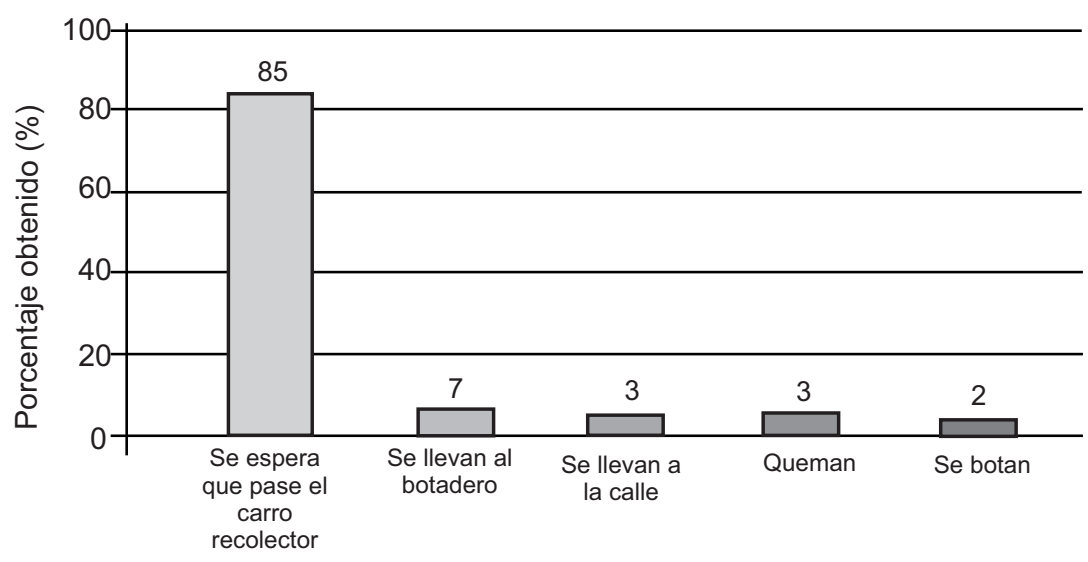

Decisiones de los pobladores

Figura 4. Evaluación de decisiones de los pobladores del Distrito

Con resultado de la encuesta representado en la Figura 4 el porcentaje de la población del distrito de Santa María se puede notar que la mayoría espera el carro recolector de residuos sólidos que nos indica que la población cada vez va

Tabla 2

La municipalidad debería mejorar el servicio del distrito incrementando su responsabilidad en una óptima disposición de su residuos domésticos, pues tiene más conciencia de la problemática medioambiental.

\begin{tabular}{lccccc}
\hline & \multicolumn{5}{c}{ Mejorar el servicio } \\
\hline & $\begin{array}{c}\text { Aumentar la } \\
\text { frecuencia de } \\
\text { recojo }\end{array}$ & $\begin{array}{c}\text { Educar a la } \\
\text { población }\end{array}$ & $\begin{array}{c}\text { Controlar al } \\
\text { personal }\end{array}$ & $\begin{array}{c}\text { Propiciar la } \\
\text { participación }\end{array}$ & $\begin{array}{c}\text { Privatizar el } \\
\text { servicio }\end{array}$ \\
\hline Porcentaje (\%) & 29,3 & 47,8 & 6,9 & 13,7 & 2,3 \\
\hline
\end{tabular}

Fuente: El autor ( 2018)

Respecto a la opinión de la población encuestada en relación al servicio municipal, se indica en la Tabla 2, que se debería educar al pueblo con $47,8 \%$, seguido de aumentar la frecuencia de recojo con 29,3\% y motivar la participación de los pobladores de Santa María con 13,7\% .

\section{DISCUSIÓN}

Con relación a la producción per cápita de Residuos Sólidos Urbanos

La generación de residuos sólidos urbanos del distrito de Santa María, como se observa en la Tabla 1, presenta una producción per cápita de $0,515 \mathrm{~kg} /$ habitante por día. Este valor sitúa al distrito de Santa María dentro del rango de producción de residuos sólidos para las ciudades de América Latina de acuerdo con Arroyo et al (1997) y Penido et al. (2006), quienes estiman que el rango de variación es de 0,370 a 1,2 $\mathrm{kg} / \mathrm{habitante} / \mathrm{día}$.

Valverde (2006) consideran que las industrias, los mercados y las malezas $u$ otros residuos vegetales provenientes de parques y jardines públicos y del barrido de las calles. Por lo que la producción per cápita será de 0,38 a 0,549 $\mathrm{kg} / \mathrm{habitante/día,} \mathrm{cuyo} \mathrm{rango} \mathrm{se} \mathrm{aproxima} \mathrm{al} \mathrm{valor} \mathrm{calculado}$ para los distritos costeros del Norte Chico del Departamento de Lima por Arnillas, Merino et al. (2009).

\section{En cuanto a su caracterización de residuos sólidos urbanos}

Los residuos sólidos urbanos de las zonas en estudio, se observa que el porcentaje más alto es de $24,75 \%$ pero sumado con otros materiales orgánicos obtuvo $42,77 \%$, en materiales inertes $22,57 \%$ y otros con menor porcentaje, estos resultados se muestra en la Tabla 2 en el grupo de residuos orgánicos y/o putrescibles (restos de cocina) y asimismo en la Figura 1 se representa en su totalidad estos tres tipos de residuos, esto se sustenta con la afirmación de Riofrío et. al. (1994) que menciona acerca de que los residuos de alimentos constituyen el componente más grande en las ciudades de los países en vías de desarrollo.

\section{Sobre la densidad promedio por día de RSU}

Encontrada en las muestras de las zonas estudiadas para el distrito de Santa María, en la Figura 2, que muestra una diferencia entre los días evaluados, tomando el valor más bajo de $88,16 \mathrm{~kg} / \mathrm{m}^{3}$ en el día jueves y el más alto de $134,66 \mathrm{~kg} / \mathrm{m}^{3}$ en el día domingo, lo que guarda relación con las afirmaciones de Flores y Villafuerte (2003); quienes señalan que las características físicas de los residuos sólidos varían en función de los hábitos de consumo y del nivel de vida de los generadores. El valor promedio de la densidad, con un valor de $110,48 \mathrm{~kg} / \mathrm{m}^{3}$, está en el intervalo de densidades de residuos sólidos considerada por Riofrío et. al. (1994) que van de 100 a $330 \mathrm{~kg} / \mathrm{m}^{3}$, correspondiente a países de ingreso medio bajo.

\section{También se evaluaron las opiniones de los pobladores.}

Dicha evaluaciones de opiniones, se recabó mediante encuestas, tal como se muestra en la figura 3; las cuales señalan que: Si con $92,3 \%$ de la población evaluada, que cree en reciclar, seguido de esta estar satisfechos con $92,0 \%$. y con $38,3 \%$, que no conocen sobre la disposición de los Residuos Sólidos domiciliarios. Se debería mejorar en cuanto a la implementación de más programa de educación ambiental, a fin de concientizar a la población; y evitar impactos ambientales negativos. Asimismo el manejo de residuos sólidos en cuanto al reciclamiento y su disposición final, esto se fundamenta con Arroyo et. al. (1997), quienes afirman que son los sectores de ingresos altos y medios los que se ven favorecidos regularmente con el mejor servicio, y en cambio los sectores pobres o marginales sólo cuentan con servicios esporádicos.

En cuanto a las evaluaciones de decisiones de los pobladores.

Con respecto a la decisión de los pobladores se obtuvo mayor porcentaje en $85 \%$ que espera que pase el carro; seguido de 
que se llevan al botadero con un resultado de $7 \%$, disminuyendo en $3 \%$ que se llevan a la calle y queman, y otro sector de la población que botan en cualquier parte en un $2 \%$ (Figura 4). Estos datos resaltan que es alentador que la población espere el carro recolector; sin embargo queda más por trabajar; por tal motivo se debe implementar programas en manejo de residuos sólidos urbanos en el cual abarque sobre educación ambiental y circuitos de recorrido del carro; ya que ciudades vecinas también esperan el carro recolector, pues según Cooperación (2008), que menciona en 13 ciudades del Norte Chico de la Provincia de Lima, en donde el 37,2 \% de la población entrevistada espera el carro recolector de residuos; puesto que la ciudad requiere con urgencia.

\section{Que debería hacer la Municipalidad}

Esta evaluación es importante, porque precisa, lo que debería realizar la Municipalidad de Santa María, en opiniones de la población señalan que la municipalidad debería educar a la población, esto lo recomienda un $47,8 \%$ de la población, por otro lado opinan un $29,3 \%$ aumentar la frecuencia de recojo, propiciar la participación $13,7 \%$, controlar a personal $6,9 \%$ o privatizar el servicio 2,3\%. En base a estos porcentajes que se precisa sobre la opinión de los pobladores de Municipalidad de Santa María. Seconcluye que se debería promover programas sobre educación ambiental, aumentar la frecuencia de recojo, fomentar la participación de los pobladores como ejes fundamentales, para de esta manera poder disminuir la contaminación ambiental y evitar su impacto ambiental negativo. Estos resultados se puede fundamentar con Glynn y Heinke (1999), quienes manifiestan que además del valor de los residuos sólidos se puede mejorar los servicios de recojo esto se a realiza en las municipales de países como Estados Unidos, Francia, Egipto, Finlandia y Australia que invierta en la educación ambiental e implementar programas en el manejo de residuos solidos.

\section{CONCLUSIONES}

La producción per cápita de residuos sólidos domiciliarios en San Bartolomé con 0,60 Kg / persona por día; seguido de Santa María con $0,58 \mathrm{Kg} /$ persona por día y Pacocha con $0,51 \mathrm{Kg}$, / persona por día.

Las zonas donde se vierten los residuos, como puntos críticos son: Pueblo Viejo, Asentamiento Humano Zapata, Lauriama, Chonta, San Bartolomé y el Asentamiento Humano Los Pinos; pues estos lugares tienen problemas en cuanto a implementación de programas de concientización ambiental para una correcta disposición final de los residuos sólidos.

Los problemas en la recolección de residuos sólidos; existe una escasa colaboración de los vecinos con $43,3 \%$ una escasa educación sanitaria con el $20 \%$, una inadecuado trabajo $13,7 \%$, no existen problemas $16,2 \%$, lo que significa que no hay un adecuado implementación de programa de concientización sobre la óptima disposición de los residuos sólidos.

\section{RECOMENDACIONES}

La Municipalidad distrital de Santa María debe adecuarse a la legislación ambiental nacional y a la vez realizar un conjunto de acciones encaminadas a lograr la máxima racionalidad en el proceso de decisión relativo a la conservación, defensa, protección y mejora del medio ambiente, basándose en una información coordinada multidisciplinaria y con colaboración de la población del distrito de Santa María

Deben impulsarse políticas ambientales para el distrito, a través de la Municipalidad o del Gobierno Regional, que estimulen la separación de residuos en la fuente, es decir, separar desde las casas los residuos que son reciclables del resto que ya no lo es; el reciclaje y la reutilización de los mismos.

Los residuos sólidos urbanos son generalmente orgánicos, por lo que es recomendable instalar una planta de reciclaje, en donde se pueda manejar adecuadamente este tipo de residuos mediante la compostificación y lombricultura, con la finalidad de obtener abonos orgánicos, como son el compost y el humus.

\section{REFERENCIAS BIBLIOGRÁFICAS}

Arnillas L., F., Merino G., M. et al. (2009), "El manejo de los residuos sólidos en los distritos costeros del norte chico del departamento de Lima (Barranca, Huaral y Huaura). Primera Edición. NOVAPRINT SAC. Lima, Perú.

Arroyo, J.; Rivas, F. y Lardinois, I. (1997), "La gestión de residuos sólidos en América Latina. IPES, ACEPESA, WASSTE. Lima, Perú.

Cantanhede, A.; Monge, G.; Sandoval, L. y Caycho, C. (2005), "Procedimientos Estadísticos para los estudios de Caracterización de Residuos Sólidos", Revista AIDIS de Ingeniería y Ciencias Ambientales: Investigación, Desarrollo y Práctica: Volumen 1, Número 1 ISSN 0718 378X, Universidad de Lima- Perú, CEPIS - Brasil. México Página 1346.

Cooperación (2008) "Conociendo la Gestión de los Residuos Sólidos en el Norte Chico". Lima, Perú. Boletín informativo disponible en la World Wide Web: http://www.cooperaccion.org.pe/publicaciones.php

Cruz N, D, (2018), "Caracterización de desechos generados por los comedores de la Universidad Nacional José Faustino Sánchez Carrión en el Distrito de Huacho". Tesis para Optar el Grado de Maestro en Ecología y Gestión Ambiental. Revista Big Bang 2018 - Volumen 7. Universidad Nacional José Faustino Sánchez Carrión. Perú. Página 50.

Cuello S. J. y Tola A. J, (1997), "Atlas mundial del medio ambiente: Preservación de la naturaleza". Editorial Cultura S.A. Madrid España Página 62 -65.

Flores, D. y Villafuerte, I. (2003). "Guía práctica No 1 para la realización de estudios de residuos sólidos domiciliarios en ciudades". Programa de Gestión Urbana de las Naciones Unidas.: IPES. Promoción del desarrollo sostenible. Quito, Ecuador.

Fuentes, C.; Carpio, J.; Prado, J. y Sánchez, P. (2008) "Gestión de residuos sólidos municipales", Gerencia para el Desarrollo 3. Ediciones ESAN (Escuela Superior de Administración y Negocios). Universidad ESAN. Lima Perú. Página 17

Glynn, H, y Heinke, G. (1999), "Ingeniería Ambiental”. Segunda Edición. Editorial Prentice-Hall. México.

Gómez, C. (2013) “III. El Desarrollo Sostenible: Conceptos Básicos, Alcance y Criterios para su Evaluación” LIBRO. Unesco. Habana-Cuba. Página 97.

INEI, (2009 - 2015) "Perú: Estimaciones y Proyecciones de Población por Sexo, según Departamento, Provincia y Distrito, 2000-2015" Boletín Especial N 18. Fondo de población de las naciones unidas, La Comisión Económica para América Latina (CEPAL) Naciones Unidas y Las acciones del Centro Latinoamericano y Caribeño de Demografía (CELADE). Lima - Perú. Página 283

Penido, M., José, H. et. al. (2006). "Manual de Gestión Integrada de Residuos Sólidos Municipales en Ciudades de América Latina y el Caribe". Instituto Brasileño de Administración Municipal. Rio de Janeiro, Brasil. 
Riofrío, G.; Oliveira, L. y Callirgos, J. (1994).” ¿Basura ó Desecho? El Destino de lo que Botamos en Lima". Centro de Estudios y Promoción de Desarrollo. Lima - Perú. Página 26.

Rodríguez L, M. (1999) "Gestión Ambiental en el Sistema de Recolección de Desechos Sólidos Urbanos en la ciudad de Trujillo". Tesis para optar el grado de Maestro en Gestión Ambiental. Escuela de Postgrado. Universidad Nacional de Trujillo. Trujillo, Perú.
Valverde G. Porfirio (2006), "Evaluación del Sistema de Recolección de Residuos Sólidos Urbanos y Propuestas de una Plan de Gestiona Ambiental en la Ciudad de Coishco. Tesis para Obtener el Grado de Maestro en Ciencias. Universidad Nacional de Trujillo, Trujillo, Perú. 